Research Article

\title{
Redox Process of Cardamom Oil in Human Blood Serum Using Cyclic Voltammetry
}

\author{
Wisam Hindawi Hoidy ${ }^{1}$, Muhammed Mizher Radhi², Mohamed Flayyh Tareef ${ }^{2}$, \\ Emad Abbas Jaffar Al-Mulla ${ }^{3}$ \\ ${ }^{1}$ Department of Chemistry, College of Education, University of Al-Qadisiyah, Al-Qadisiyah City, Iraq. \\ ${ }^{2}$ Radiological Techniques Department, Health and Medical Technology College-Baghdad, Middle Technical University (MTU), Iraq. \\ ${ }^{3}$ College of Health and Medical Techniques, Al-Furat Al-Awsat Technical University, 54003 Al-Kufa, Iraq. \\ \Corresponding author. E-mail: wisam.hoidy@qu.edu.iq
}

Received: Dec. 12, 2019; Accepted: Mar. 16, 2020; Published: Mar. 17, 2020.

Citation: Wisam Hindawi Hoidy, Muhammed Mizher Radhi, Mohamed Flayyh Tareef, and Emad Abbas Jaffar Al-Mulla, Redox Process of Cardamom Oil in Human Blood Serum Using Cyclic Voltammetry. Nano Biomed. Eng., 2020, 12(I): 99-103.

DOI: 10.5101/nbe.v12i1.p99-103.

\begin{abstract}
Cardamom oil is one of the active herbs used as a treatment in different diseases by the function of the blood serum components. Among the methods used for identification of function group which acted in the human body, the method of electrochemical analysis using cyclic voltammetry was used to determine the effectiveness of cardamom by the oxidation-reduction reaction in the blood medium. Results showed that the cardamom compound presented peaks of oxidation-reduction in the blood serum medium of 0.8 and $-0.65 \mathrm{~V}$, respectively. The oxidation peak disappeared in alkaline $\mathrm{pH}$; hence, the cardamom acted as anti-oxidative compound in alkaline medium and with ascorbic acid. But the oxidation peak was enhanced in acidic $\mathrm{pH}$. Other electrochemical parameters of cardamom in blood serum medium were determined, including studies on different scan rates, reliability and stability.
\end{abstract}

Keywords: Cyclic voltammetry; Cardamom oil; Ascorbic acid; Redox reaction; Blood serum

\section{Introduction}

As in the study of the electrochemical analysis of pharmaceutical compounds, researchers have been presently studying medicinal herbs and their effects on the composition of blood by oxidation-reduction reactions [1-6].

Cardamom is an herb, whose seeds and oils from the seeds are used in the manufacture of the drug as shown in the chemical composition in Fig. 1. Cardamom is used for digestive problems including heartburn, intestinal cramps, irritable bowel syndrome, diarrhea, constipation, complaints of liver and gallbladder, and loss of appetite. It is also used to treat colds, other infections, cough, bronchitis, stomatitis, urinary tract problems, epilepsy, headache and high blood pressure [7].

Many biochemical studies have already shown that cardamom has properties that improve health, and this corresponds to the typical properties of agate. Although it is a very promising compound for the feeding field, there is a lack of studies regarding the properties of electrolysis. Cardamom was studied in the voltammetric method. The quantitative limit of the proposed voltmeter methodology is sufficient to analyze the effect of this compound on several phytochemical matrices [8]. Because of its antioxidant and anti-inflammatory quality, spice intake helps to treat diseases., and cardamom is one of these spices. Therefore, The objective of this research was to 


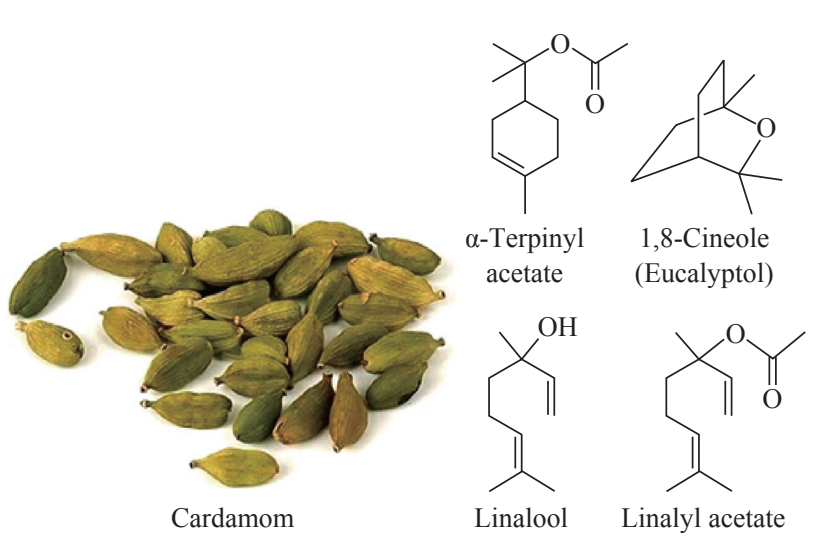

Fig. 1 Structure of cardamom.

determine the effect of cardamom supplementation on blood lipids, glycemic indexes and blood pressure in pre-diabetes women. Green cardamom supplementation may have a protective effect on HDL-C level in prediabetic subjects. It improves some blood parameters in these subjects. However, its effects do not differ from placebo [9].

During distillation, cardamom components are released from the seeds based on their variability. This research examined cardamom oil collection in different fractions. Sensory, physical and chemical analyses, including spectral mass analysis and gas chromatography, showed a clear fractionation of the components during distillation, helping the end user to choose the correct fraction based on which cardamom oil was to be used [10].

Cardamom oil components showed variations in composition in quality and quantity. The study of GC and GC-MS resulted in 55 compounds being identified, representing $98 \%$ of the total oil [11].

Individual antioxidants in spices (thymol, precious acids, capsaicin, rosemary, and eugenol) are irreversibly oxidized at $0.88-1.25$ volts in a carbon electrode in $0.1 \mathrm{~mL}$ of lithium solution in ethanol. A method for determining the voltmeter of the spices' antioxidant amplitude (AOC) based on their antioxidant oxidation was developed. AOC decreased in "clove series $>$ juniper berries $>$ nutmeg $>$ cinnamon rosemary $>$ anise $>$ black thyme $>$ black pepper, ginger, basil $>$ turmeric $>$ red pepper leaf bay leaf $\geq$ coriander sweet red pepper $>$ cumin" [12].

Cardamom belongs to the chemical category of chalcones, a highly important phenolic compound due to its health-promoting properties. Electrochemical behavior on a suspended mercury electrode was compared with chalcone via cyclic voltammetry measurement $(\mathrm{CV})$. Electrochemical reductions of cardamom are usually an irreversible reaction. It seemed that all alternatives owned by cardamom were stabilizing, making it difficult to reduce the occurrence of the compound [13].

In this work, cardamom oil was studied of its electrochemical properties in blood medium to discover the effect of the oil on the blood components by its different $\mathrm{pH}$ and other voltammetric properties of redox reactions.

\section{Experimental Cyclic voltammetric technique}

The measurements were carried out using the EZstat (Potentiostat / Glvanostat) series from NuVant Systems (USA). The electrochemical analysis cell was connected to the potentiostat and controlled for cyclic voltammetric measurement $(\mathrm{CV})$ by the software installed on the computer. Silver / silver chloride (Ag/ $\mathrm{AgCl}$ in $3 \mathrm{M} \mathrm{KCl}$ ) was used as a reference electrode and as a counter electrode platinum wire $(1 \mathrm{~mm}$ diameter). In this study, the glass working carbon electrode (GCE) was used after cleaning by polishing and treatment for measuring performance using ultrasonic waterways for $10 \mathrm{~min}$.

\section{Materials}

Cardamom oil was received from Abideen (Pakistan). Blood samples from healthy humans obtained from the Baghdad Medical Center were collected for analysis after the serum was completely separated from the blood by an electronic centrifuge of type 8-1 (3,000 cycles / min). Deionized water was used to prepare water solutions. All samples of blood serum were diluted by 1: $9 \mathrm{~mL}$ (serum : deionized liquid) with deionized water. In a cyclic voltammetric cell, $10 \mathrm{~mL}$ of dilute serum was placed it. Glassy carbon electrode was used as a working electrode (GCE) after performing by polishing and cleaning the surface of the electrode as show in Fig. 2.

\section{Procedure}

The potentiostat was used in this study to determine the oxidation-reduction reaction of cardamom in $10 \mathrm{~mL}$ of blood serum samples and placing the 3 electrodes (working, reference and counter electrodes) in the solution of the voltammetry cell which connected the three electrodes in the potential station then with personal computer to provide the cyclic voltammogram. 


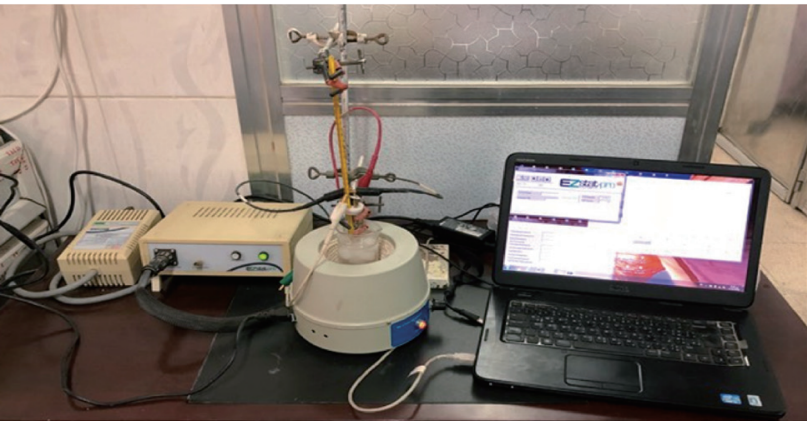

Fig. 2 Cyclic voltammetry parts.

\section{Results and Discussion}

Blood serum based cardamom oil was studied to reveal electrochemical properties at different $\mathrm{pH}$, scanning rates, ascorbic acid concentration and reliability (stability) by the oxidation-reduction reaction in blood serum samples as shown in the Scheme 1.<smiles>CC(=O)OC(C)(C)C1CC=C(C)CC1</smiles>

Scheme 1 The oxidation-reduction reaction of cardamom oil.

\section{Effect of different $\mathrm{pH}$}

Cardamom oil was studied in different $\mathrm{pH}$ of blood serum medium to find the electrochemical properties of oxidation-reduction current peaks of the oil, which had oxidation and reduction current peaks in the medium of neutral blood serum $(\mathrm{pH}=7)$ at 0.85 and $-0.6 \mathrm{~V}$ as shown in Fig. 2. On the other hand, the reaction in alkaline blood medium had different property that the alkaline $\mathrm{pH}$ acted in an anti-oxidative effect of cardamom oil on the blood serum component, disappearing the oxidation current peak with enhanced the reduction current peak as shown in Fig. 2 [15].

Fig. 3 illustrated the electrochemical property of cardamom in blood serum medium as oxidative stress in acidic blood serum medium compared with that in neutral medium $(\mathrm{pH}=7)$, which enhanced both oxidation-reduction current peaks. These results were concluded in Fig. 4, comparing the electrochemical behavior of cardamom in alkaline and in acidic blood serum medium. Fig. 5 and 6 illustrate the cardamom oil at different $\mathrm{pH}$ of blood serum medium for oxidation-

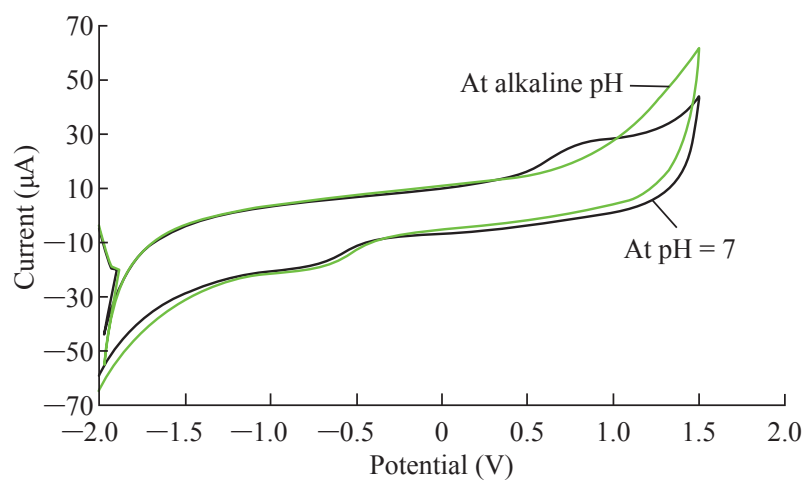

Fig. 3 The cyclic voltammogram of cardamom oil in different blood $\mathrm{pH}$ (neutral and alkaline.

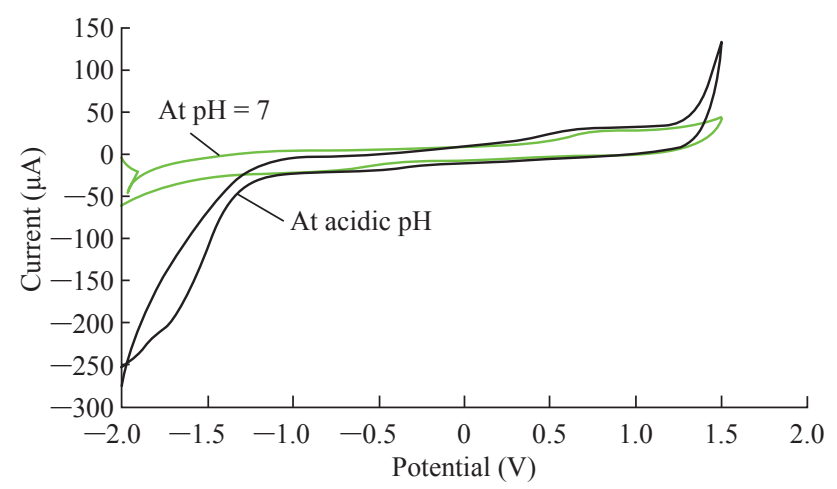

Fig. 4 The cyclic voltammogram of cardamom oil in different blood $\mathrm{pH}$ (neutral and acidic).

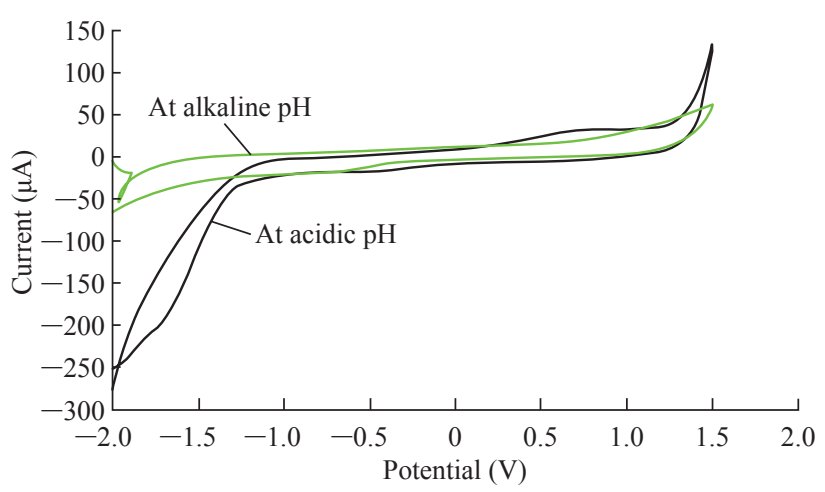

Fig. 5 The cyclic voltammogram of cardamom oil in different blood $\mathrm{pH}$ (alkaline and acidic).

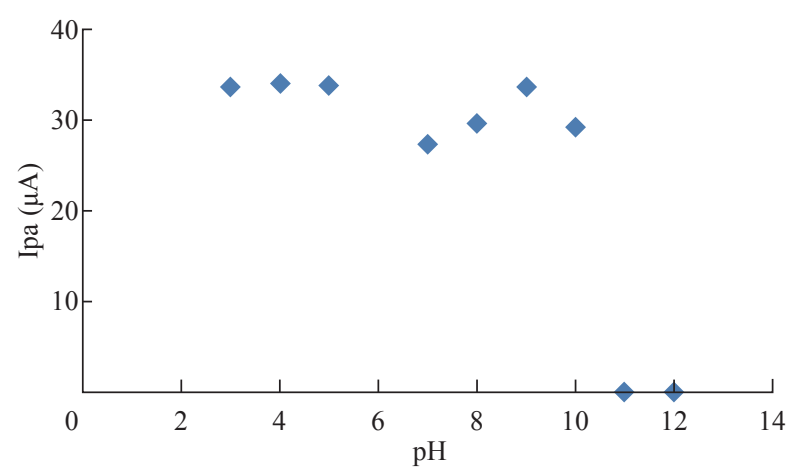

Fig. 6 The relationship between oxidation current peaks of cardamom oil in different $\mathrm{pH}$ of blood medium. 
reduction current peaks [16].

\section{Effect of different scan rates}

It was found from the results that cardamom oil acted as antioxidant reagent in blood serum medium by disappearing the oxidation current peak and enhancing the reduction current peak, which had a good electrochemical behavior of increasing the scan rate against the cathodic current peak as shown in Fig. 7, with the relationship $y=98.788 x+47267$ (Fig. 8) and a good sensitivity $\mathrm{R} 2=0.9844$. The relationship in Fig. 8 was indicated by the slope of the graph, which differed significantly from the theoretical value of the half of the diffusion control process (the theoretical value being 50), indicating the presence of a compound in the blood serum medium [17].

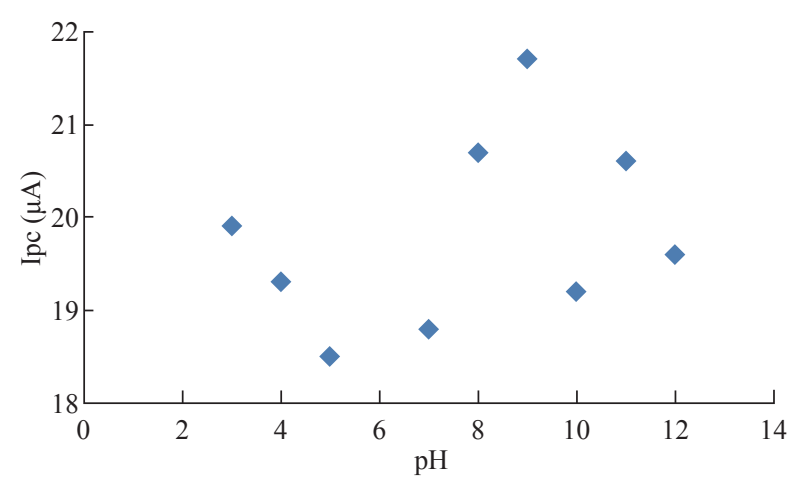

Fig. 7 The relationship between oxidation current peaks of cardamom oil in different $\mathrm{pH}$ of blood medium.

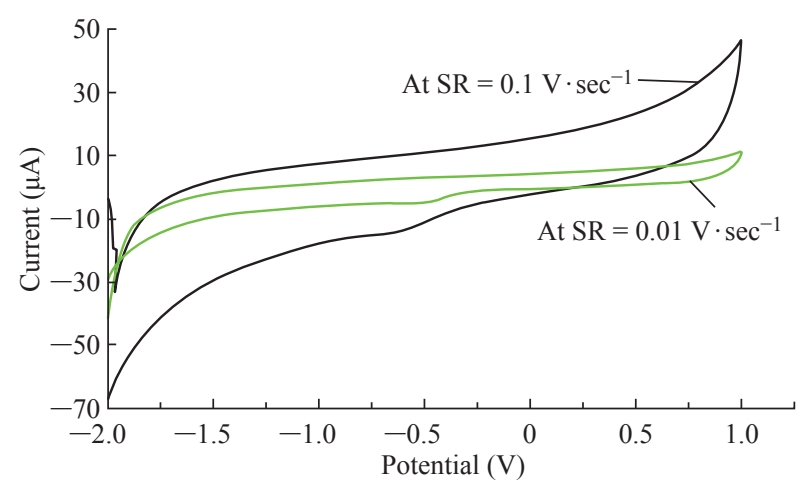

Fig. 8 The cyclic voltammogram of blood cardamom oil at various scanning frequencies $(0.01-0.1 \mathrm{~V} / \mathrm{sec})$.

\section{Effect of ascorbic acid}

Fig. 9 illustrates the study of ascorbic acid's effect on the cardamom oil in blood serum medium. It is known that ascorbic acid presents alkaline behavior in blood serum medium [18]. So, ascorbic acid acts as antioxidant reagent in blood serum medium with cardamom oil.

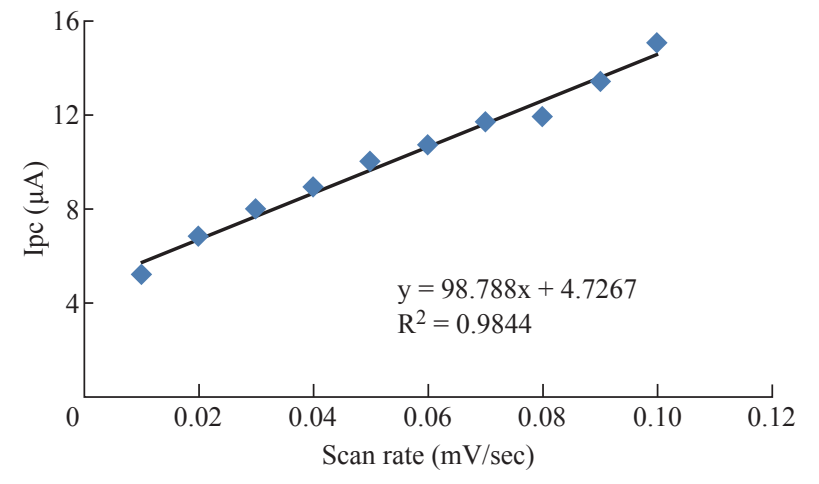

Fig. 9 Plotting of reduction current peak of cardamom oil in alkaline blood medium against different scan rates.

\section{Reliability and stability}

Cardamom oil compound in blood serum medium was studied by glassy carbon electrode in cyclic voltammetry. 10 times of scanning the cyclic voltammetry was studied as shown in Fig. 10 and 11 , and the relative standard deviation (RSD) was determined for reduction current peak at $-0.65 \mathrm{~V}$ of the oil with value $\pm 0.3 \%$, which had a good reliability and stability of the oil in blood serum medium with these results [19].

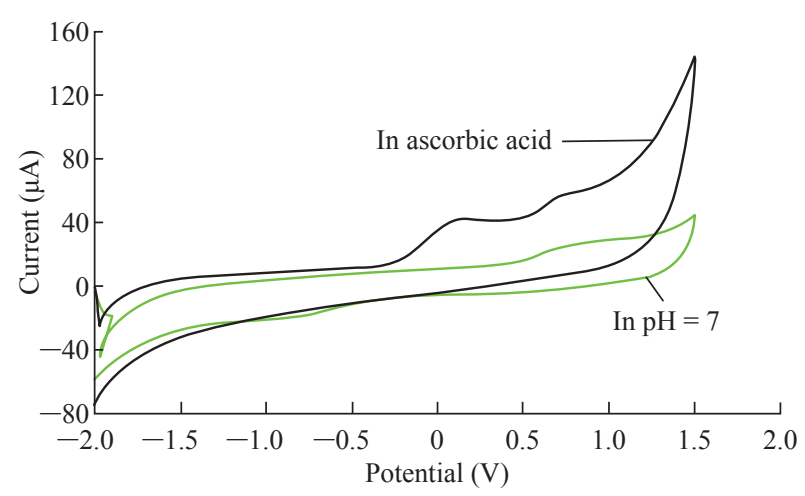

Fig. 10 The cyclic voltammogram of cardamom oil with ascorbic acid in the blood at specific pH using GCE as the working electrode and $\mathrm{Ag} / \mathrm{AgCl}$ as the SR reference electrode of $0.1 \mathrm{~V} / \mathrm{sec}$.

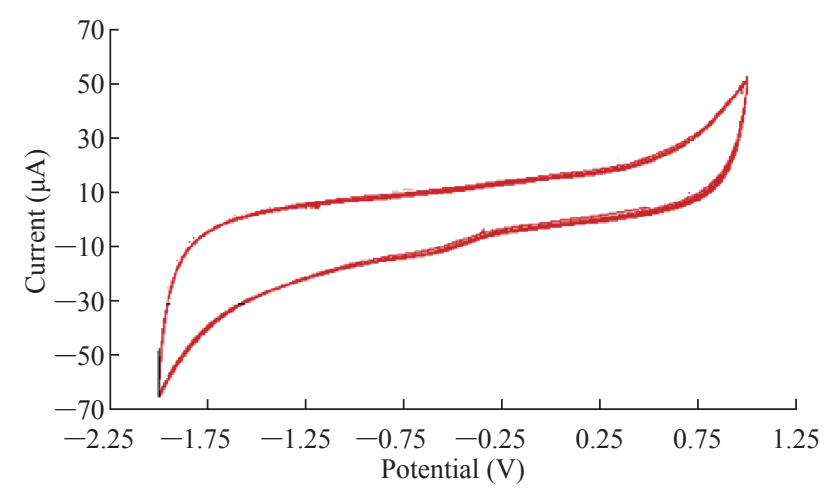

Fig. 11 The cyclic voltammogram of cardamom oil in blood medium at ten times using GCE as working electrode and Ag/ $\mathrm{AgCl}$ as reference electrode at $\mathrm{SR}$ of $0.1 \mathrm{~V} / \mathrm{sec}$. 


\section{Conclusions}

Cardamom oil compound was studied in blood serum medium by cyclic voltammetric technique using glassy carbon electrode to find the electrochemical behavior of the oil in blood serum medium with and without ascorbic acid. It was found that the oil compound could be considered as anti-oxidative reagent in blood serum medium which enhanced cathodic current peak at $-0.65 \mathrm{~V}$ in alkaline $\mathrm{pH} 11$ according to the cyclic voltammogram. The study indicated that the oil compound was a good antioxidant reagent in blood serum medium and affected ascorbic acid on the reduction current peak as antioxidant reagent effect which enhanced the reduction current peak.

\section{Conflict of Interests}

The authors declare that no competing interest exists.

\section{References}

[1] M.M. Radhi, H.N. Abdullah, M.S. Jabir, et al., Electrochemical effect of ascorbic acid on redox current peaks of $\mathrm{CoCl}_{2}$ in blood medium. Nano Biomed Eng, 2017, 9(2): 103-106.

[2] M.M. Radhi, A.A.A. Albakry, A.M. Jassim, et al., Electrochemical study of $\mathrm{Pb}(\mathrm{II})$ in present of each ascorbic acid, glucose, urea and uric acid using blood medium as an electrolyte. Nano Biomed Eng, 2016, 8(1): 9-15.

[3] M.M. Radhi, S.A. Alassady, N.T. Barakat, et al., Electrochemical oxidation effect of each ascorbic acidand folic acid on lead ions in human blood. International Journal of Pharmacy and Chemistry, 2017, 3(3): 33-40.

[4] M.M. Radhi, Z.N. Hamed, S.S. Ezzaldeen, et al., Effect of micro- and nanoparticles of ampicillin trihydrate on blood medium: A voltammetric study. Nano Biomed Eng, 2017, 9(3): 185-190.

[5] M.M. Radhi,, Y.K. Al-Haidarie, and A.I. Ibrahim, The electrochemical effect of different temperatures on sodium saccharine in blood medium using modified working electrode CNT/GCE by cyclic. Journal of Silicate Based and Composite Materials, 2017, 69(1): 24-28

[6] Y.K. Abdul-Amir, M.M. Radhi, and E.A.J. Al-Mulla, Use of nano-sensors of the interferences between $\mathrm{Pb}$ ((II) with each of $\mathrm{Mg}(\mathrm{II}), \mathrm{Zn}(\mathrm{II}), \mathrm{Mn}(\mathrm{II}), \mathrm{Ca}(\mathrm{II}), \mathrm{Co}(\mathrm{II})$ and PO4-3 in blood medium: An electrochemical study. Nano Biomed
Eng, 2017, 9(3): 199-207.

[7] F. Anwar, A. Abbas, K.M. Alkharfy, et al., Cardamom (Elettaria cardamomum Maton) oils. Essential oils in food preservation, flavor and safety. Amsterdam: Academic Press, 2015: 295-301.

[8] A.M. Carvalho, L.M. Gonçalves, I.M. Valente, et al., Analysis of cardamonin by square wave voltammetry. Phytochem Anal, 2012, 23(4): 396-399.

[9] Y. Fatemeh, F. Siassi, A. Rahimi, et al., The effect of cardamom supplementation on serum lipids, glycemic indices and blood pressure in overweight and obese prediabetic women. J Diabetes Metab Disord, 2017, 16: 40.

[10] B.K. Mani, V. Murthy, M. Boland, et al., Analysis of constituents in different Fractions collected during distillation of Cardamom oil for flavour and fragrance applications. J Appl Pharm Sci, 2017, 7 (1): 177-183.

[11] R. Joshi, P. Sharma, V. Sharma, et al., Analysis of the essential oil of large cardamom (Amomum subulatum Roxb.) growing in different agro-climatic zones of Himachal Pradesh India. J Sci Food Agric, 2013, 93(6):1303-1309.

[12] G.K. Ziyatdinova, H.C. Budnikov, Evaluation of the antioxidant properties of spices by cyclic voltammetry. $J$ Anal Chem, 2014, 69(10): 990-997.

[13] E.M.Tavares, A.M.Carvalho, L.M.Gonçalves, et al., Chemical sensing of chalcones by voltammetry: Transchalcone, cardamonin and xanthohumol. Electrochim Acta, 2013, 90(15): 440-444.

[14] M. Przygodzka, D. Zielińska, Z. Ciesarovác, et al., Comparison of methods for evaluation of the antioxidant capacity and phenolic compounds in common spices. LWT - Food Sci Techno, 2014, 58(2): 321-326.

[15] M.M. Radhi, I.N. Khelkal, E.N. Naji, et al., Electrochemical characterization of micro- and nanoparticles of ceftriaxone in human blood serum samples using cyclic voltammetry. Journal of Silicate Based and Composite Materials, 2019, 71(2): 54-58.

[16] C. Engin, S. Yilmaz, G. Saglikoglu, et al., Electroanalytical investigation of paracetamol on glassy carbon electrode by voltammetry. Int J Electrochem Sci, 2015, 10: 1916-1925.

[17] M.M. Radhia, E.A.J. Al-Mulla, Voltammetric characterization of grafted polymer electrode selfmodification with carbon nanotubes (GPESMCNT). Portugaliae Electrochimica Acta, 2016, 34(2): 97-103.

[18] J. Sochor, J. Dobes, O. Krystofova, et al., Electrochemistry as a tool for studying antioxidant properties. Int $J$ Electrochem Sci, 2013, 8: 8464-8489.

[19] L.D. Torbeck, Statistical solutions: \%RSD: Friend or foe. Pharmaceutical Technology, 2010, 34(1).

Copyright $@$ Wisam Hindawi Hoidy, Muhammed Mizher Radhi, Mohamed Flayyh Tareef, and Emad Abbas Jaffar AlMulla. This is an open-access article distributed under the terms of the Creative Commons Attribution License, which permits unrestricted use, distribution, and reproduction in any medium, provided the original author and source are credited. 